\title{
NUMERICAL SIMULATION OF FLOW IN A FUEL-INJECTOR OF AN AIRCRAFT ENGINE COMBUSTOR USING BUILDING-CUBE METHOD
}

\author{
Younghwa Cho' ${ }^{1}$, Rahul Bale ${ }^{2}$, Makoto Tsubokura ${ }^{3}$, and Nobuyuki Oshima ${ }^{4}$ \\ ${ }^{1}$ School of Engineering, Hokkaido University \\ Kita 13, Nishi 8, Kita-ku, Sapporo, Hokkaido 060-8628, Japan \\ E-mail: younghwa@eis.hokudai.ac.jp, URL: https://www.eng.hokudai.ac.jp/english/ \\ ${ }^{2}$ RIKEN Center for Computational Science (R-CCS) \\ 7-1-26 Minatojima-minami-machi, Chuo-ku, Kobe, Hyogo 650-0047, Japan \\ E-mail: rahul.bale@riken.jp, URL: https://www.r-ccs.riken.jp/en/ \\ ${ }^{3}$ RIKEN Center for Computational Science (R-CCS) \\ 7-1-26 Minatojima-minami-machi, Chuo-ku, Kobe, Hyogo 650-0047, Japan \\ E-mail: mtsubo@riken.jp, URL: https://www.r-ccs.riken.jp/en/ \\ ${ }^{4}$ School of Engineering, Hokkaido University \\ Kita 13, Nishi 8, Kita-ku, Sapporo, Hokkaido 060-8628, Japan \\ E-mail: oshima@eng.hokudai.ac.jp, URL: https://www.eng.hokudai.ac.jp/english/
}

Keywords: Building-Cube Method, Immersed Boundary Method, Grid-Dependency, Aircraft Engine Combustor.

\begin{abstract}
In this study, we investigate grid dependency on local mesh refinement for the numerical simulation of cold flow in an aircraft engine's fuel-injector. The numerical simulation of fully compressible Navier-Stokes equations is conducted using a hierarchical Cartesian mesh-based solver known as "CUBE". Using the results of the high-resolution simulation as the basis, the gird dependency analysis is carried out. In addition, we evaluate the weak scaling of the underlying solver.
\end{abstract}

\section{INTRODUCTION}

The geometry of an aircraft engine's industrial-scale fuel-injector is considerably sophisticated, and computer-aided design (CAD) data are usually burdened with geometric imperfections: 'dirtiness.' This makes a mesh generation be an extremely time-consuming task. Furthermore, the computational load of the numerical mesh generation becomes enormous to resolve geometric complexities and boundary layers appropriately. required to resolve geometric complexities and boundary layers is enormous. Therefore, it is necessary to develop strategies for easing mesh generation and computational load. Accordingly, to ease the mesh generation and immersed boundary method (IBM) for handling complex non-watertight 
geometries, we adopt a hierarchical Cartesian meshing technique known as the building-cube method (BCM). Focusing on the cold flow simulation of a fuel-injector of an aircraft engine, this study presents an investigation on grid dependency; here, the cold flow simulations are conducted by a fully compressible flow solver of a BCM-based numerical framework named "CUBE". In addition, the scalability performance of CUBE is evaluated by a massively parallel computer system "FUGAKU" and it exhibits optimal scalability.

\section{NUMERICAL FRAMEWORK}

\subsection{Building-Cube Method}

The numerical framework adopted in this study is based on a solver known as CUBE, which is built on a Cartesian meshing methodology known as BCM [1, 2]. In BCM, the computational domain is discretized into cubic blocks referred to as cubes. In addition, finer cubes can be populated in the region of interest, such as the target geometry. In this study, the refinement ratio of the size of adjacent cubes with different sizes is restricted to two. Each cube in the domain is subdivided into cubic cells such that all cubes have the same number of cells. Because a cube is handled as the fundamental unit of the mesh data structure, the computational cost of every cube is identical, thus making the domain decomposition straightforward. A schematic representation of the discretization procedure using BCM is presented in Figure $\mathbf{1}$.
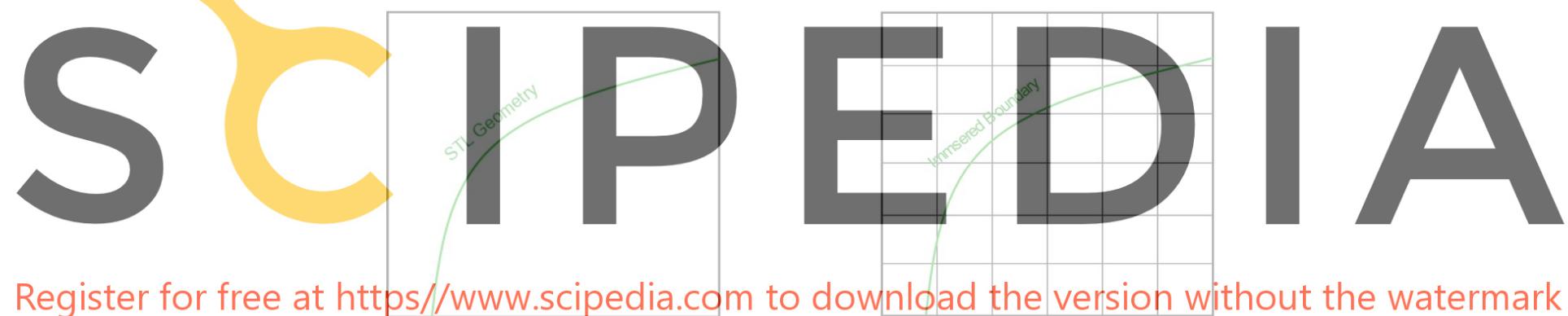

Register for free at https//www.scipedia.com to download the version without the watermark

(a) STL geometry in computational domain.

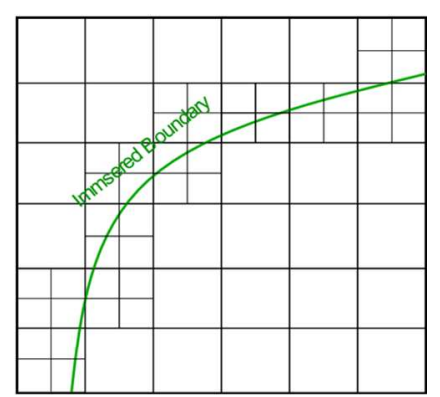

(c) Subdivision of cubes in the domain. (b) Boundary immersed into cubes.

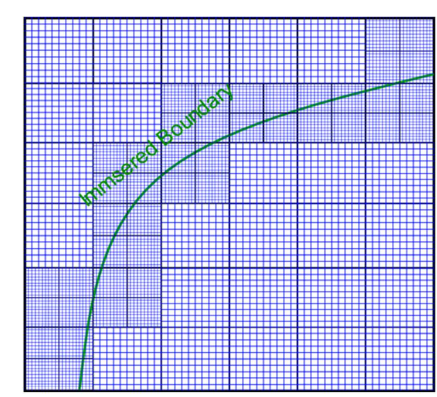

(d) Cell (blue) generation in each cube.

Figure 1: Schematic of discretization procedure using BCM 


\subsection{Immersed Boundary Method}

The geometry of an aircraft engine's fuel-injector is considerably sophisticated, and the industrial-scale CAD data are usually non-watertight. Many IB methods that can be found in the literature are generally not well-suited for treating non-watertight CAD data. For such IBMs, additional time is required for preprocessing the data. However, the IBM developed by $\mathrm{Li}$, et al. [3] addresses this limitation and allows CAD geometry to be non-watertight, thereby eliminating the effort required for preprocessing. In this work we empoy the IBM of $\mathrm{Li}$, et al..

\subsection{Governing Equations}

In this study, we adopt fully compressible Navier-Stokes equations, and the compact form is expressed as:

$$
\frac{\partial Q}{\partial t}+\nabla \cdot F=0
$$
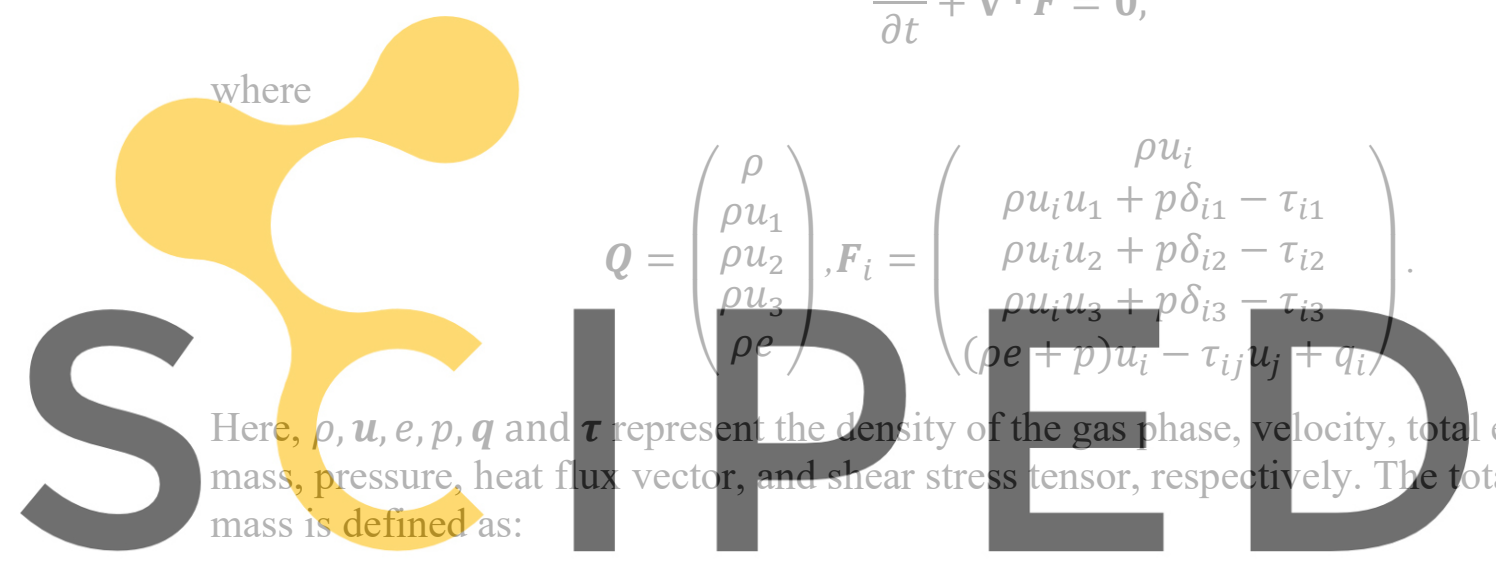

1

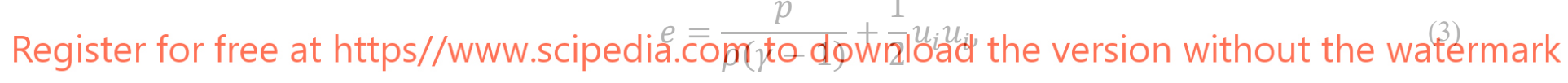

where $\gamma$ is the specific heat ratio. The heat flux $q$ is given by:

$$
\boldsymbol{q}=-\lambda \boldsymbol{\nabla} T
$$

where $\lambda$ and $T$ represent the thermal conductivity and temperature, respectively. In addition, the shear stress tensor $\boldsymbol{\tau}$ is given as:

$$
\tau_{i j}=\mu\left(2 S_{i j}-\frac{2}{3} \delta_{i j} \frac{\partial u_{k}}{\partial x_{k}}\right)
$$

where $\boldsymbol{S}$ is the strain-rate tensor expressed as:

$$
S_{i j}=\frac{1}{2}\left(\frac{\partial u_{j}}{\partial x_{i}}+\frac{\partial u_{i}}{\partial x_{j}}\right) .
$$

Finally, the discretization methodologies are presented in Table 1. The simulation adopted in this study is a large-eddy simulation with an implicit sub-grid scale (SGS) model. Here, the numerical dissipation added through the Roe-upwinding scheme is assumed to function as a 
proxy for the SGS model, while the grid size serves as the spatial filter.

Table 1: Discretization methodologies adopted in this study

\begin{tabular}{cc}
\hline Time Marching & $\begin{array}{c}\text { Low-Mach Preconditioned Lower-Upper Symmetric Gauss-Seidel (LU- } \\
\text { SGS) with Solution Limited Time-Stepping (SLTS) }[4,5,6]\end{array}$ \\
\hline $\begin{array}{c}\text { Advective Flux } \\
\text { Term }\end{array}$ & Low-Mach Preconditioned Roe with 5th-Order MUSCL [4, 7, 8] \\
\hline $\begin{array}{c}\text { Diffusive Flux } \\
\text { Term }\end{array}$ & 2nd-Order Central Difference \\
\hline
\end{tabular}

\section{INVESTIGATION OF GRID DEPENDENCY}

\subsection{Geometry of Reference Fuel-Injector}

The reference fuel-injector, an ECO-SGS type fuel-injector, was provided by Kawasaki Heavy Industries, Ltd. (KHI), and the CAD data (STL format) are presented in Figure 3-(a). In addition, the diameter $D$ of the fuel-injector, axial length $L$, and width of the air-slit nozzle $W$ are approximately $62[\mathrm{~mm}], 60[\mathrm{~mm}]$, and 1 [mm], respectively.

There are three types of paths in the pilot nozzle as illustrated in Figure 3-(b). Furthermore, the inflow-plane is arranged to introduce inflow boundary conditions, and the reference fuelinjector is combined with a duct to guide the influx of flow from the inflow-plane. The

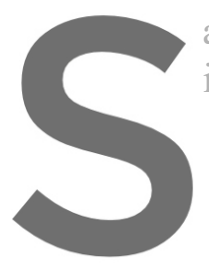
arrangement of the geometries, as is presented in Figure 3-(c).

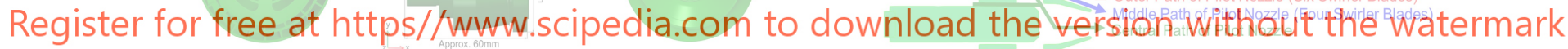
(a-1) Behind view $\quad(a-2)$ Side view $\quad$ (a-3) Front view

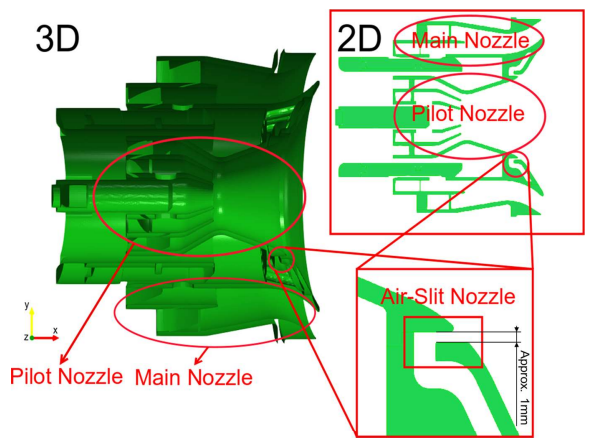

(a-4) Cross section

(a) CAD data of referee fuel-injector
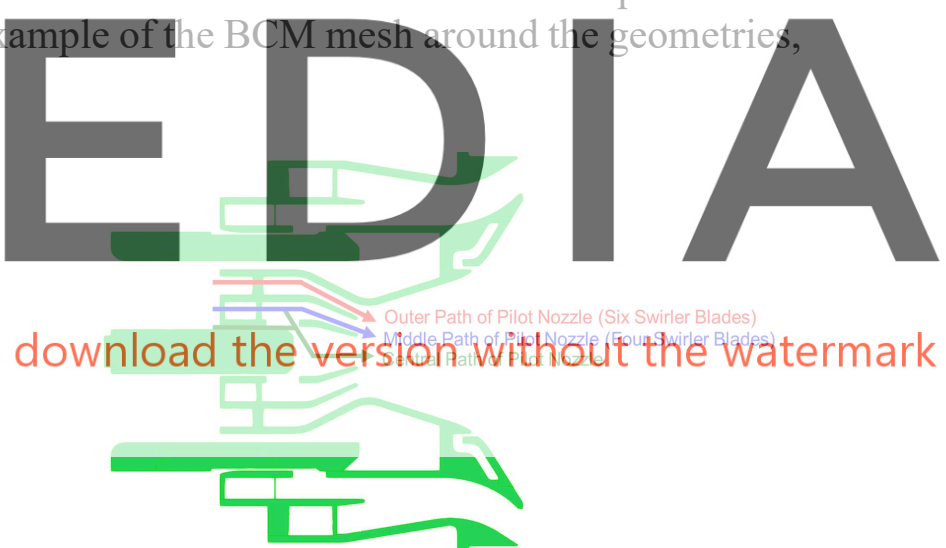

(b) Paths of pilot nozzle

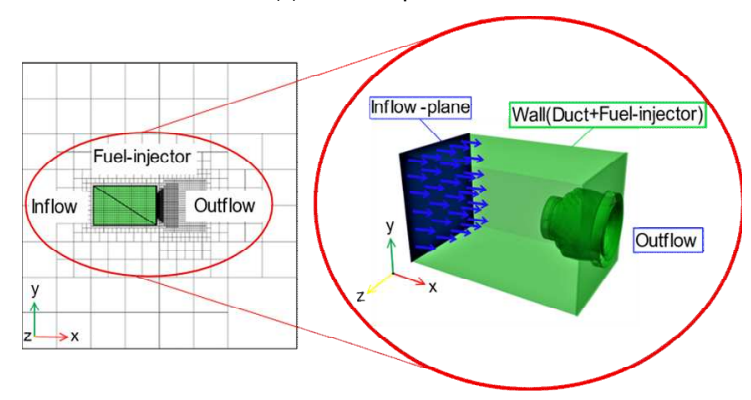

(c) Arrangement of geometries and BCM meshes

Figure 3: CAD data of referee fuel-injector and the arrangement of the geometries in the computational domain 


\subsection{Reference Experimental Condition and Computational Setup}

In this study, an operational condition of the experiment conducted by Kawasaki Heavy Industries, Ltd. (KHI) is referenced to establish the computational setup of this study. The experimental condition is presented in Table 2.

Table 2: Reference experimental condition, provided by KHI

\begin{tabular}{cc}
\hline Ambient Pressure & $1[\mathrm{~atm}]$ \\
\hline Ambient Temperature & $288.15[\mathrm{~K}]$ \\
\hline Inflow Mass Flow Rate & $0.074[\mathrm{~kg} / \mathrm{s}]$ \\
\hline Inner Pressure of Duct & $1.05[\mathrm{~atm}]$ \\
\hline
\end{tabular}

In the computational domain, no-slip, and adiabatic conditions are applied to the wall. The velocity applied in the inflow-plane is estimated such that the inflow mass flow rate becomes the same value presented in Table 2, whereas the velocity applied on the inflow-plane is $\mathbf{u}=$ $(5.376,0,0)[\mathrm{m} / \mathrm{s}]$. In addition, the initial condition is tabulated in Table 3 . Density is calculated using the equation of state for ideal gas from the ambient temperature and pressure presented in Table 2.

Table 3: Initial condition applied to the entire domain this study

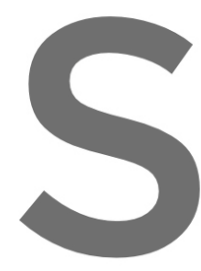

\subsection{Meshes}

For the investigation on grid dependency, mesh spacing of 0.095 [mm] and mesh size of 2.
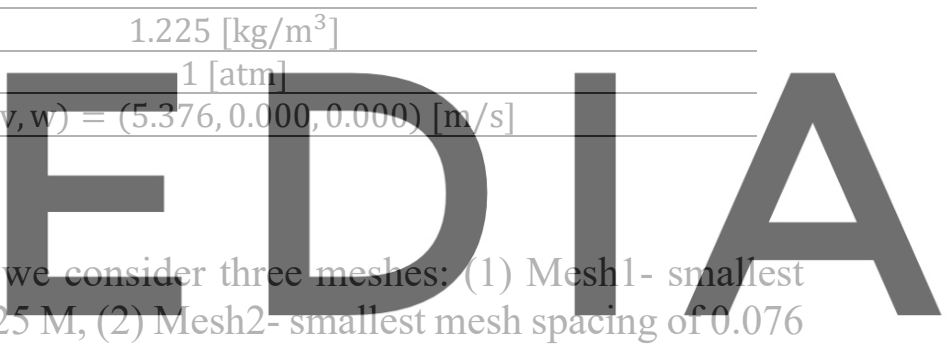

[mm] and mesh size of $120 \mathrm{M}$, and (3) Mesh3- smallest mesh spacing of 0.050 [mm] and mesh

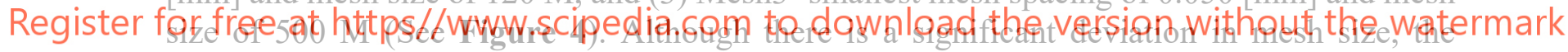

difference in the time consumed for the mesh generation is negligible, and it is approximately within $0.5[\mathrm{~h}]$.

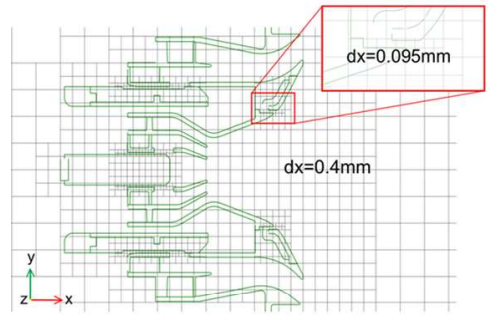

(a) Mesh1 (Size: 25,000,000)

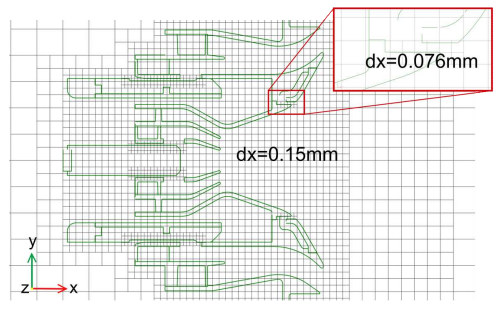

(b) Mesh2 (Size: 120,000,000)

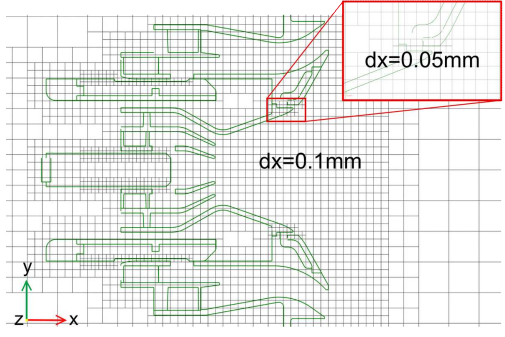

(c) Mesh3 (Size: 500,000,000)

Figure 4: Meshes used for the grid dependency investigation 


\subsection{Results}

\subsubsection{Results of Inner Pressure of Duct}

First, the inner pressure transition of the duct is illustrated in Figure 5. In the range of 01000-time steps (: shaded part), the profile exhibits initial transiency. Therefore, in this simulation, the average in time is considered in the range of 2000-4000-time steps, where the profile is stationary, and the averaged inner pressure of the duct is presented in Figure 6. As presented in Figure 6, the inner pressure of duct becomes smaller as the grid becomes finer, and it converges to the referenced condition presented in Table 2. For Mesh3, the averaged pressure exhibit reasonable value within $1 \%$ of the reference value. However, because the difference between Mesh2 and Mesh3 is still noticeable, we need to examine more refined case for checking the convergence.
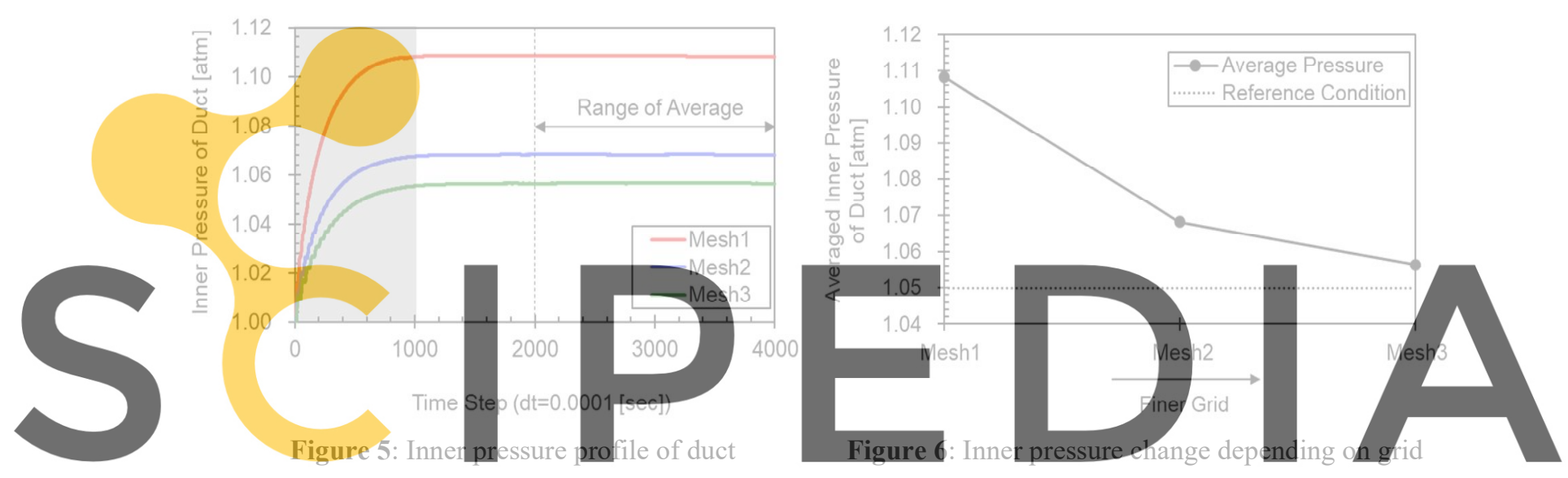

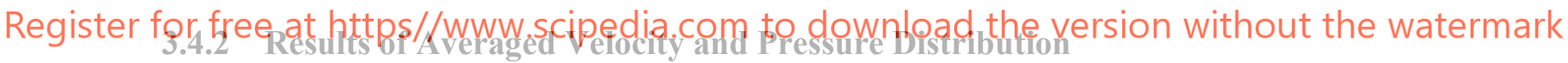

\subsubsection{Comparison of Miesh 1 and viesh3}

As illustrated in Figure 4, in Mesh1, the finest resolution of $d x=0.095[\mathrm{~mm}]$ is assigned in the narrowest parts represented by the air-slit nozzle, thereby making Mesh1 approximately two times coarser than Mesh3.

The results of the simulation, where Mesh1 was adopted, is presented in Figure 7-(a). This indicates that although Mesh1 was two times coarser than Mesh3, it was able to resolve the flow through the air-slit nozzle. However, in the region of flow from pilot nozzle (A-region), a significant difference between Mesh1 and Mesh3 is depicted in Figure 7-(c). In the Mesh3 case, the flow from the pilot nozzle (A-region) is attached to the wall. However, in the Mesh1 case, its counterpart is developed as jet flow, separating it from the wall. Therefore, the Aregion near the exit of the pilot nozzle appears to be a susceptible region that significantly influences the flow. In contrast, A-region of Mesh3 exhibits negative pressure. The negative pressure region is considered to be established by the flow attached to the wall, and because the flow is not attached to the wall in Mesh1, the A-region does not exhibit negative pressure.

Another difference in flow between Mesh1 and Mesh3 indicates the difference in the 
distribution of velocity magnitude in the B- and C-regions. The velocity magnitude in B-region of Mesh1 is larger than its Mesh3 counterpart. Contrastively, the velocity magnitude in Cregion of Mesh1 is smaller than its Mesh3 counterpart. Owing to the small magnitude of velocity, it can be stated that the swirl from the pilot nozzle is incompletely developed in the Mesh1 case. In addition, the pressure distribution in the B- and C-regions indicates that the pressure gradient of Mesh3 becomes more moderate than its Mesh1 counterpart. This suggests that the resolution of Mesh1 in the region is insufficient such that more pressure gradients were required to pass through the region, especially the outer path of pilot nozzle (Figures 3-(b) and 7-(a)). Therefore, it can be stated that because most of the flow of the pilot nozzle passed through the central path, where the swirl effect does not appear, the swirl in the A-region became weak; hence, no negative pressure appeared in the A-region, and jet flow was developed.

In summary, Mesh1 was successfully able to resolve all flow-paths in the geometry. However, the result is remarkable because the flow and pressure distribution in the A-region (pilot region) do not qualitatively agree with its counterpart in Mesh3. Consequently, it is inferred that the pilot region exhibits the highest dependency upon resolution change.

\subsubsection{Comparison of Mesh2 and Mesh3}

As illustrated in Figure 5, in Mesh2, the resolution of the air-slit nozzle is selected to be $d x=0.076[\mathrm{~mm}]$. As is evident from the analysis of the results obtained from Mesh1, the pilot region is highly sensitive to resolution. Therefore, for the present mesh, i.e., Mesh2, a mesh spacing of $0.15[\mathrm{~mm}]$ is

The simulation result from the result that Me pilot nozzle. The velocity with its Mesh3 counterparts. In addition, the distri ore, for the pros
gion, which is
opted, is presen
e to capture th
ution of air-slit $0.4[\mathrm{~mm}]$ in Mesh 1 .
ted in Figure 7 -(b). I
flow attached to the
nozzle exbribit good It is evident
e wall of the
d agreement
the $B$ - and C-regions is also considerably consistent with its counterpart in Mesh3. However, focusing on

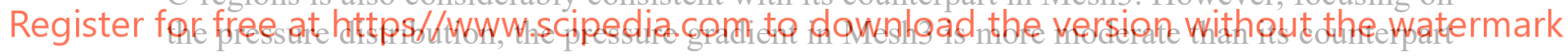

in Mesh2, and the A-region of Mesh3 exhibits a stronger negative pressure than its Mesh2 counterpart. Considering that the pressure gradient along the flow demonstrates the flow patin's resistance, the following can be stated: because, in Mesh3, the outer path of the pilot nozzle (Figure 3-(b)) exhibits more moderate pressure gradient than that of Mesh2 (Figures 7-(b-2, c-2)), more flow passes through the outer path, and a stronger swirl is can be formed in the pilot nozzle. It is considered that this stronger swirl contributes to the stronger negative pressure in the A-region of Mesh3. In addition, another difference between Mesh2 and Mesh3 can be found in Figure 8. In the Mesh2 case (Figure 8-(a)), the swirl flow from the middle path of the pilot nozzle, which has four swirler blades, appears significantly in the plane of $x=0[\mathrm{~m}]$, although it does not appear on the outer path of the pilot nozzle, which has 6 swirler blades. However, in the Mesh3 case (Figure 8-(b)), because the swirl from the outer path becomes more significant, and that from the middle path becomes dissipated by joining the outer swirl, the swirl from the outer path significantly appears in the plane of $x=0[\mathrm{~m}]$. This indicates that Mesh2 was unable to accurately reproduce the 3D structure of the swirl in pilot region (A- and C-regions). Accordingly, it can be inferred that the pilot region still exhibits grid dependency between Mesh2 and Mesh3. 
Based on the results obtained from Mesh1, Mesh1 was able to identify the key regions that significantly influence the flow, and generate a new mesh accordingly- Mesh2. Therefore, regarding the A-, B-, and C-regions illustrated in Figure 7, Mesh2 obtained results consistent with those of Mesh3. However, as shown in Figure 8, Mesh2 was actually unable to accurately reproduce the 3D structure of the swirl in the pilot region. Therefore, in future works, it is necessary to conduct this study using finer grid instead of Mesh3.

\section{WEAK SCALING EVALUATION}

In this evaluation, we solely consider the fully compressible Navier-Stokes solver. The evaluation was conducted using meshes whose size are 1179648, 9437184, 75497472, 603979776 and 3505070080 , whereas the simulations were carried out using 192, 1536, 12288, 98304 and 576000 CPUs, respectively. Here, the speedup ratio is defined as:

$$
\text { Speedup Ratio }=\frac{\text { Runtime-Per-Timestep of Basis Mesh } \times \frac{\text { Target Mesh Size }}{\text { Basis Mesh Size }}}{\text { Runtime-Per-Timestep of Target Mesh }}
$$

In this study, the basis mesh is set as the smallest mesh with a size of 1179648 . The weak scaling results are plotted in Figure 9, and the simulation exhibits excellent scalability in the range of the set of mesh-CPUs considered in this study. In addition, the parallel efficiency at 576000 CPUs (mesh size: 3505070080 ) is $75.5 \%$.
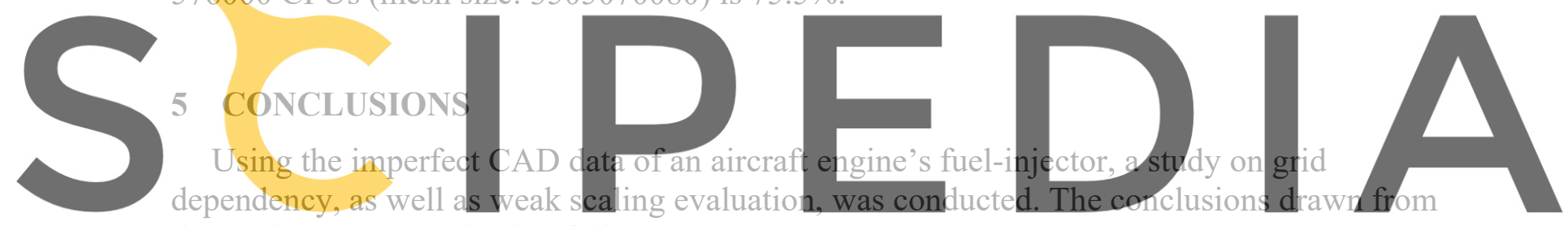

the study are summarized as follows.

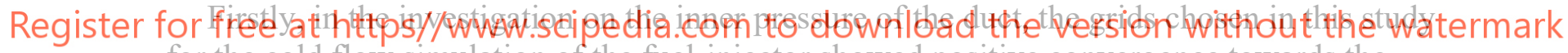

for the cold flow simulation of the fuel-injector showed positive convergence towards the

reference experimental duct pressure. While the agreement between the pressure of mesh 3

and reference value is acceptable, it may be possible to improve the results marginally using a more refined mesh.

Also, between Mesh2 and Mesh3, the velocity and pressure distribution agreed well in

Figure 7. However, as shown in Figure 8, the 3D structure of the pilot region's swirl differed between Mesh2 and Mesh3. Thus, it can be said that the most sensitive region was the pilot region (A- and $\mathrm{C}$-regions). In particular, the paths that include the swirler (i.e., the outer and middle paths illustrated in Figure 3-(b)) function as key regions of the grid dependency. As a future work, it is necessary to investigate more refined (larger) cases than Mesh3 to study the mesh dependency.

Finally, through the weak scaling evaluation, it has been demonstrated that the present simulation with CUBE exhibits optimal parallel efficiency with approximately seven times larger cases than Mesh3, which is the largest mesh size used in the present study on grid dependency. Therefore, even in more refined cases, the effective parallel computation can also be expected. 


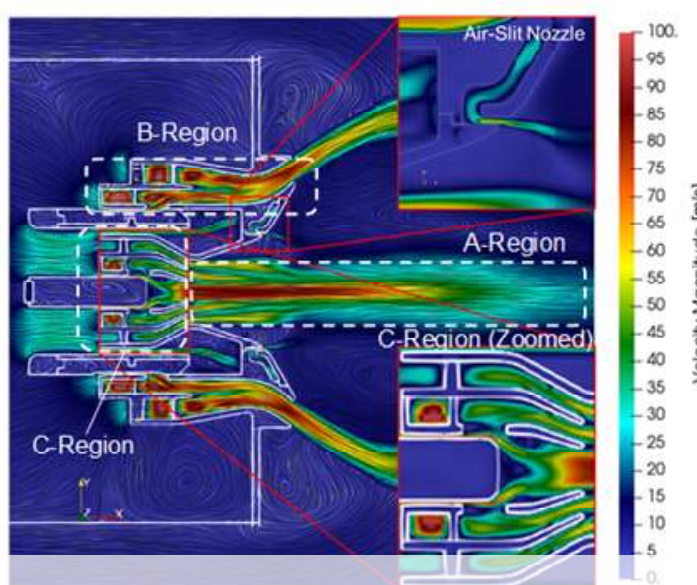

(a-1) Velocity distribution of Mesh1
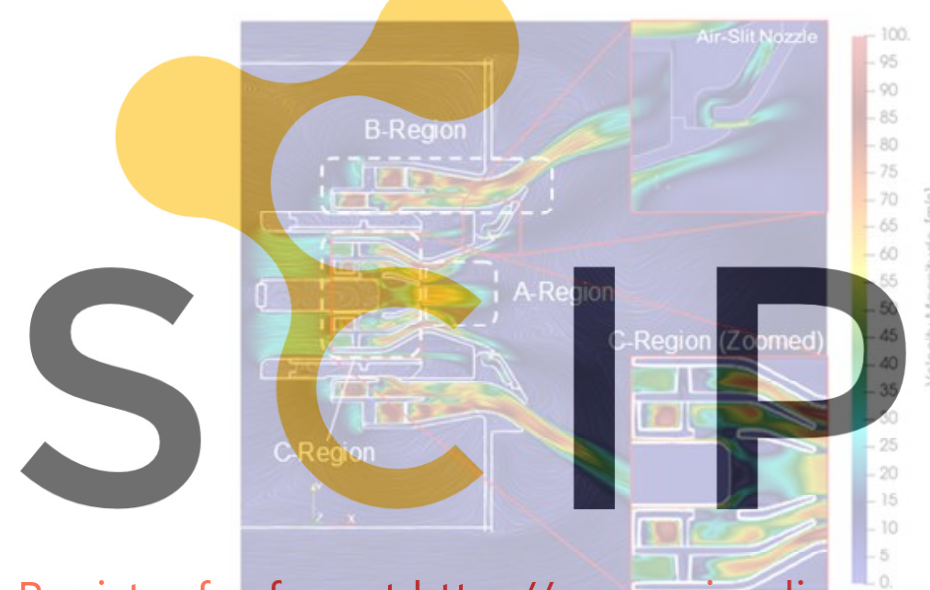

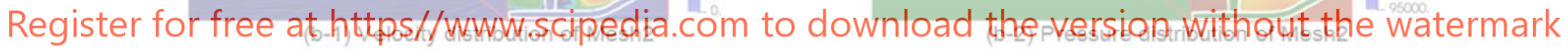

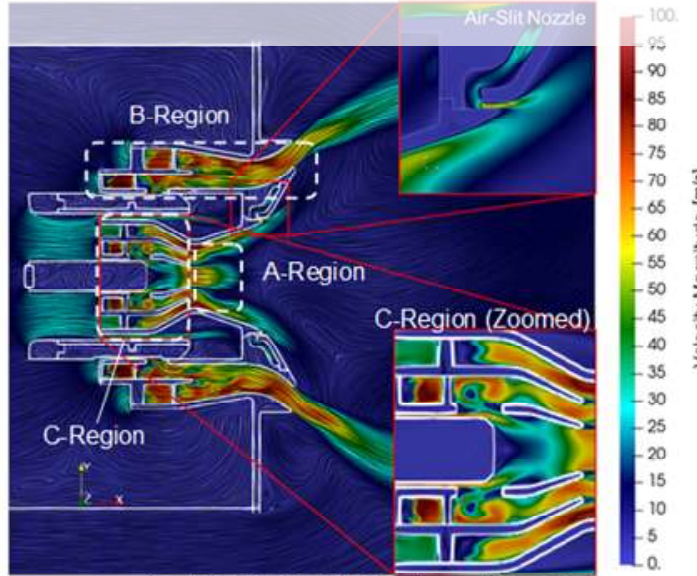

(c-1) Velocity distribution of Mesh3

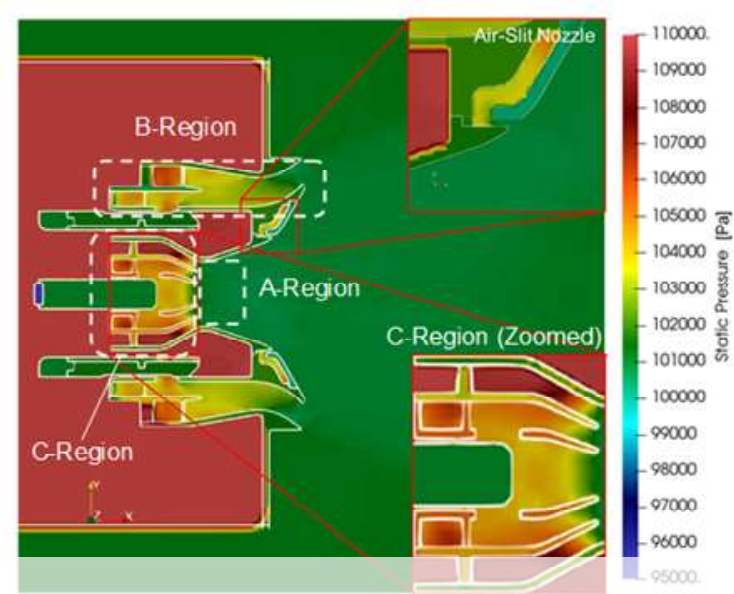

(a-2) Pressure distribution of Mesh1

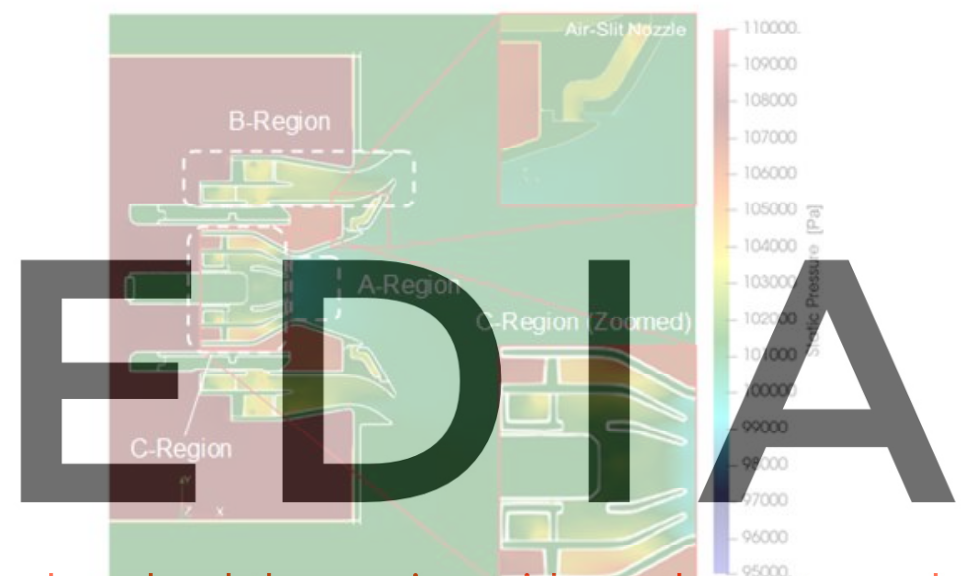

Figure 7: Averaged velocity and pressure distribution of each mesh 

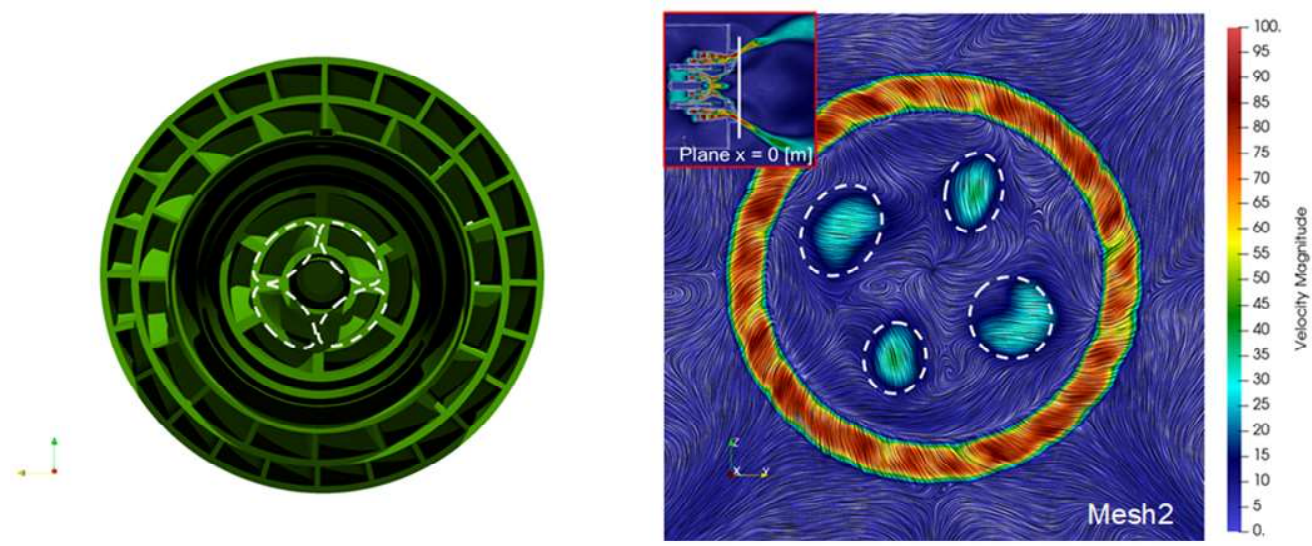

(a) Number of blades in middle path of pilot nozzle (left) and velocity distribution of mesh2 at plane $x=0[\mathrm{~m}]$ (right)
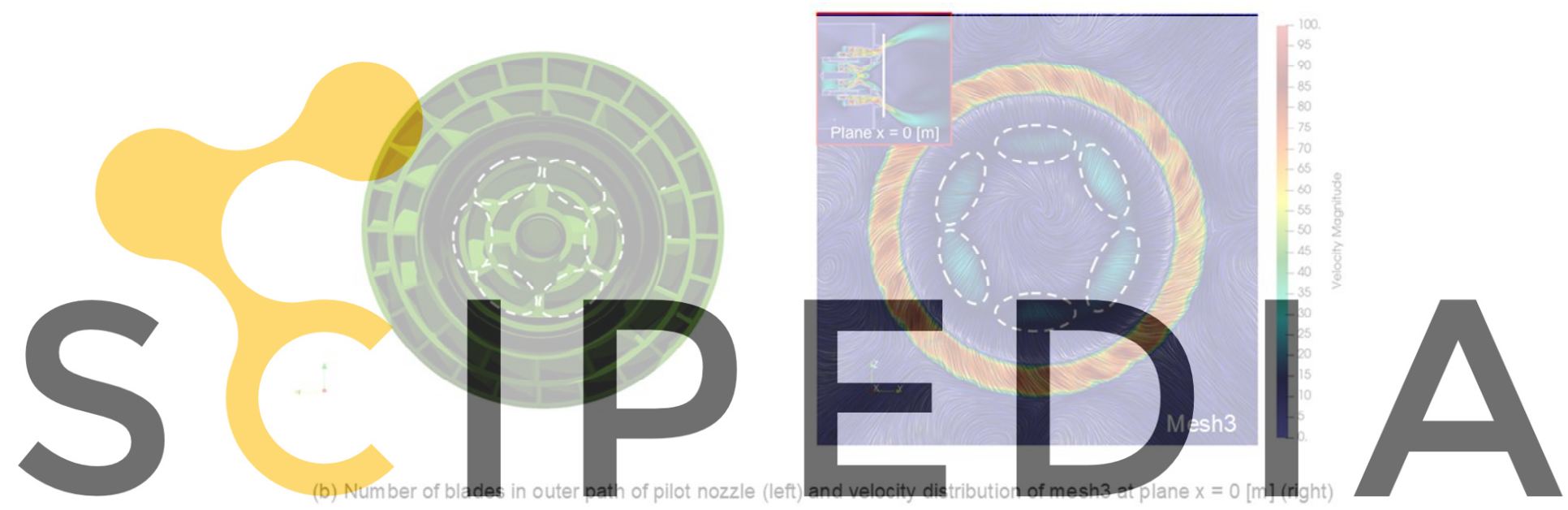

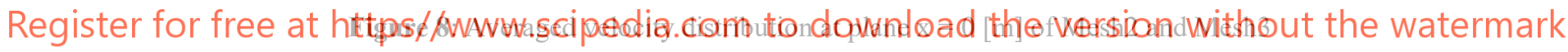

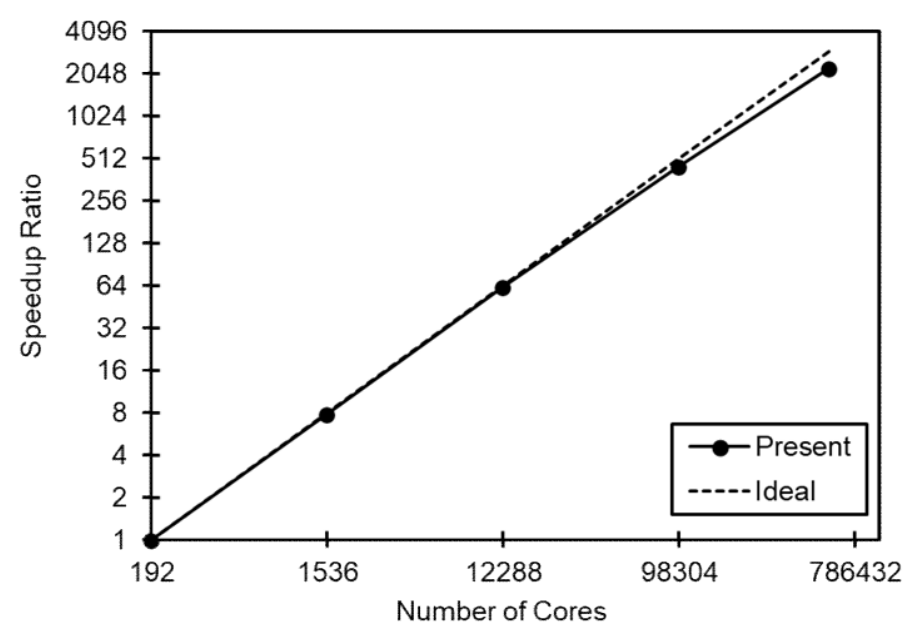

Figure 9: Speedup ratio against the number of CPUs of a fully compressible flow simulation 


\section{ACKNOWLEDGEMENT}

This work was carried out under a collaboration between Hokkaido University, RIKEN, Center for Computational Science, and Kawasaki Heavy Industries, Ltd.. This work employed computational resources of the supercomputer, "FUGAKU", provided by RIKEN via the HPCI System Research Project (Project ID: hp200160) and supercomputer "ITO" provided by the Research Institute for Information Technology, Kyushu University (Project ID: hp200017), as well as the supercomputer "Grand Chariot" provided by the Information Initiative Center, Hokkaido University, Japan. This work was also supported by $f^{3}$ Engineering Education and Research Center, Faculty of Engineering, Hokkaido University, Japan. Finally, Kenta Takami, an undergraduate student at Hokkaido University, is acknowledged for research assistance.

\section{REFERENCES}

[1] JANSSON, Niclas, et al. CUBE: A scalable framework for large-scale industrial simulations. The international journal of high performance computing applications, 2019, 33.4: 678-698.

[2] NAKAHASHI, Kazuhiro. Building-cube method for flow problems with broadband characteristic length. In: Computational fluid dynamics 2002. Springer, Berlin, Heidelberg, 2003, 77-81.

[3] LI, Chung-Gang; TSUBOKURA, Makoto; BALE, Rahul. Framework for simulation of natural convection in practical applications. International Communications in Heat and Mass Transfer, 2016, 75: 52-58.

[4] WEISS, Jonathan M.; SMITH, Wayne A. Preconditioning applied to variable and constant density flows. AIAA journal, 1995, 33.11: 2050-2057.

[5] YOON, Seokkwan; JAMESON, Antony. Lower-upper symmetric-Gauss-Seidel method for the Euler and Navier-Stokes equations. AIAA journal, 1988, 26.9: 1025-1026.

[6] LIAN, Chenzhou; XIA, Guoping; MERKLE, Charles L. Solution-limited time stepping to enhance reliability in CFD applications. Journal of Computational Physics, 2009, 228.13: 4836-4857.

[7] ROE, Philip L. Approximate Riemann solvers, parameter vectors, and difference schemes. Journal of computational Physics, 1997, 135.2: 250-258.

[8] KIM, Kyu Hong; KIM, Chongam. Accurate, efficient and monotonic numerical methods for multi-dimensional compressible flows: Part II: Multi-dimensional limiting process. Journal of computational physics, 2005, 208.2: 570-615. 